Fußball-Europameisterschaft und nachhaltige Entwicklung

\section{Nachhaltigkeit im Abseits?}

Die Dimensionen von medial vermarkteten Großveranstaltungen nehmen stetig zu. Und mit ihnen auch die Auswirkungen. Neben den ökonomischen Effekten rücken dabei auch ökologische und soziale Auswirkungen in den Fokus. Die Fußball Europameisterschaft 2008 ist ein Beispiel, wie in Zukunft die Effekte auf nachhaltige Entwicklungsprozesse evaluiert werden können. Von Christian Moesch

$S^{\text {e }}$ it dem Brundtlandbericht der Weltkommission für Umwelt und Entwicklung vor rund zwanzig Jahren wird der Nachhaltigkeitsbegriff in den verschiedensten Bereichen verwendet und regelmäßig in die öffentliche Diskussion gebracht. Vor dem Hintergrund immer stärker spürbarer Umweltprobleme gewinnen die Idee der Nachhaltigkeit und die damit verbundenen Handlungsweisen auch im Bereich des Event-Managements zunehmend an Aktualität und Dringlichkeit. So haben auch SportMega-Events sich vermehrt an den Anforderungen der Nachhaltigkeit zu orientieren.

Neben sportspezifischen Kriterien müssen der Umweltverantwortung, der Sozialverträglichkeit sowie der wirtschaftlichen Ergiebigkeit spezielle Beachtung geschenkt werden. Ziel ist es, dass die Durchführung des Anlasses die intraund intergenerationelle Solidarität nicht gefährdet. Im Sinne der Nachhaltigkeit soll jene Lebensqualität gefördert werden, die mit geringerem Einsatz an nicht vermehrbaren Ressourcen sowie abnehmender Belastung der Umwelt und der Menschen erzielt werden kann (Müller 2003).

\section{Langfristige Effekte der EURO 2008}

Im Auftrag des Schweizerischen Bundesamtes für Sport werden die Effekte der Fußball-Europameisterschaft 2008 (EURO 2008) auf die nachhaltige Entwicklung in der Schweiz untersucht. Um die längerfristigen Konsequenzen und die eventspezifische Dynamik der EURO 2008 aufzeigen zu können, umfasst die Studie eine Zeitspanne von fünf Jahren. Zudem wird versucht, die Erkenntnisse für kommende Events nutzbar zu machen und eine Messmethode zu entwickeln, die längerfristige Vergleiche ermöglicht.

Dies stellt insofern eine Herausforderung dar, als es kaum möglich ist, die Wirkungen derartiger einmaliger Events auf die nachhaltige Entwicklung umfassend abzubilden. Die Wissenschaft nähert sich dieser Fragestellung an, indem sie sich auf die Messung zentraler Effekte im komplexen Nachhaltigkeitsnetzwerk konzentriert. In diesem Fall orientierte sich die Auswahl der über 20 Kernindikatoren einerseits an bereits durchgeführten vergleichbaren Untersuchungen anderer Sport-MegaEvents sowie an aktuellen Fragestellungen(Bieger 2003; Rahmann 1998).

\section{Sind Verhaltensänderungen möglich?}

Grundsätzlich ist die Begleitstudie auf die ganze Schweiz ausgerichtet. Verschiedene in der Studie untersuchte Effekte werden aber für die vier Schweizer Austragungsregionen Basel, Bern, Genf und Zürich differenziert erfasst, womit eine gewisse interregionale Vergleichbarkeit gewährleistet wird. Die Erhebung der einzelnen Indikatoren findet vor, während und nach dem Event statt. Der Schluss- bericht ist auf Sommer 2009 terminiert. Es wäre wünschenswert, eine noch längere Zeitperiode $\mathrm{zu}$ berücksichtigen, jedoch ergibt sich aus wissenschaftlicher Sicht das Problem, dass bereits kurze Zeit nach dem Event eine starke Überlagerung durch andere Einflussfaktoren stattfindet. Somit wäre eine eindeutige Zuordnung der Effekte mit steigender Dauer nach Ende der Veranstaltung zunehmend schwieriger.

Bezüglich der ökologischen Dimensionen wird die Abfallentsorgung evaluiert, das Verkehrsaufkommen untersucht sowie der Energie- und Wasserverbrauch gemessen. Im Zentrum steht natürlich die Frage, ob die EURO längerfristige Veränderungen im Verhalten erwirken kann, beispielsweise bezüglich der Nutzung des öffentlichen Verkehrs. Trotz des KombiTickets, einem mit dem Stadioneintritt verknüpften 36-Stunden-Generalabonnement, ist dies für die Schweiz eher unwahrscheinlich, denn derartige Verhaltensänderungen hängen primär von Impulsen in der Angebotsgestaltung ab. Diese sind aber im Zusammenhang mit der EURO nicht vorgesehen. Zudem ist die Schweiz bereits heute bekannt für eine ausgeprägte Kultur des öffentlichen Verkehrs. Nachhaltige Verbesserungen sind eher durch verbesserte Öko-Effizienz im Rahmen von Zertifizierungen und Prozessoptimierungen zu erwarten.

Ein spezielles Augenmerk wird der Mobilität der Funktionäre in der Vor- und Nachbereitung geschenkt. Seit dem Jahr 2005 werden bei den wichtigsten Akteuren in der Schweiz die aufgrund der EURO zurückgelegten Verkehrswege erhoben. Bis Ende 2007 wurden bereits über 5,5 Millionen Personenkilometer erfasst. Drei Viertel davon entfallen auf den Flugverkehr, während der motorisierte Individualverkehr und die Bahn ungefähr gleichwertig genutzt wurden.

\section{Die Einstellung der Bevölke- rung als Indikator}

Zu den zentralen Nachhaltigkeitsindikatoren gehört auch die Einstellung der Schweizer Bevölkerung zum Mega-Event. Bereits im Herbst 2005 wurde die Schwei- 
zer Bevölkerung diesbezüglich erstmals befragt. Eine zweite Befragungswelle folgte im Oktober 2007. Zwischen den beiden Messpunkten haben sich keine substantiellen Veränderungen ergeben (Rütter 2007a). Beide Befragungen registrierten ein großes Wohlwollen quer durch alle Bevölkerungsschichten und Regionen.

Unmittelbar nach der EURO wird nochmals nach derselben Methode befragt, wodurch die Veränderung in der Wahrnehmung offengelegt werden soll. Eine erfolgreiche Durchführung der EURO 2008 könnte sich beispielsweise in einer nachhaltigen Veränderung der generellen Einstellung gegenüber Sportgroßveranstaltungen niederschlagen. Damit würde auch die Diskussion um Olympische Winterspiele in der Schweiz wieder neu genährt.

\section{Nicht messbare Wirkungen}

Im Brennpunkt der bisherigen Evaluation standen die kurz- und langfristigen volkswirtschaftlichen Effekte. Die Perspektivstudie hat ergeben, dass kurzfristig eine Wertschöpfung von 640 bis 860 Millionen Schweizer Franken erwartet werden kann (Rütter 2007b). Trotz Multiplikatorenwirkung werden diese Effekte nur über einen kurzen Zeitraum spürbar sein. Längerfristig sind die intangiblen Effekte ausschlaggebend, ob die Volkswirtschaft nachhaltig positiv beeinflusst wird. Die stärkste Wirkung erhofft man sich von der Image- und Werbewirkung einer erfolgreichen Durchführung. Umgekehrt besteht natürlich auch die Gefahr, dass Pleiten und Fehler während des Events negative Attribute transportieren. Der Einfluss der EURO 2008 auf die Entwicklung des Images der Schweiz wird in Deutschland und in Frankreich im Zeitraum 2007 bis 2009 durch drei Befragungswellen untersucht.

Einzelne intangible Wirkungen, wie die Begeisterung, der Erlebnisnutzen oder der Spaßwert, die zumindest für den Fußballfan zentrale Bedeutung haben, sind nur schwer zu erfassen und lassen sich kaum quantifizieren. Eine abschließende Beurteilung der eventspezifischen Wirkungen auf die nachhaltige Entwick- lung wird erst bei Abschluss der Untersuchung im Sommer 2009 möglich sein.

\section{Literatur}

Bieger, T. / Müller, HR. / Elsasser, H.: Nachhaltigkeit der FIS alpinen Ski WM 2003 in St. Moritz. Schlussbericht. St. Moritz 2003.

Müller, H.R.: Tourismus und Ökologie. 2. Auflage. Wechselwirkungen und Handlungsfelder. München 2003

Rahmann, B. / Weber, W. / Groening, Y. / Kurscheidt, M. / Happ, H.G. / Pauli, M.: Sozioökonomische Analyse der Fußball-Weltmeisterschaft 2006 in Deutschland. Köln 1998.

Rütter, H. / Müller, HR. / Stettler, J.: Zweite Bevölkerungsbefragung zur Akzeptanz, Einstellung und Bewertung der UEFA EURO 2008. Rüschlikon 2007a.

Rütter, H. / Müller, HR. / Stettler, J.: Wirtschaftliche Wirkungen der UEFA EURO 2008TM in der Schweiz. Aktualisierung der Studie von 2004. Rüschlikon 2007b.

I AUTOR + KONTAKT

Christian Moesch ist wissenschaftlicher Mitarbeiter an der Universität Bern und Koordinator des Forschungsprojektes „UEFA EURO 2008TM - Evaluation und Effekte auf die nachhaltige Entwicklung".

Universität Bern, Forschungsinstitut für Freizeit und Tourismus (FIF), Schanzeneckstrasse 1, 3001 Bern, Schweiz. Tel.: +41 31631 33-85, E-Mail: christian.moesch@fif.unibe.ch

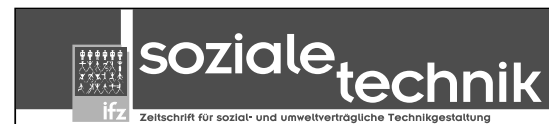

Seit 17 Jahren ist SOZIALE TECHNIK - herausgegeben vom Interuniversitären Forschungszentrum für Technik, Arbeit und Kultur (IFZ) - ein Forum für die interdisziplinäre Technikforschung und liefert Beiträge für eine sozial- und umweltverträgliche Gestaltung von Technologien. SOZIALE TECHNIK erscheint viertelï̈hrlich, ein Jahresabonnement kostet $€ 18$,- (für Studierende $€ 13$,-).

Kostenloses Probeabonnement!

\section{Mit Beiträgen zu:}

Technologie \& Politik

- Umwelt \& Energie

$\square$ Neve Biotechnologien

$\square$ Frauen \& Technik

口 Informations- \&

Kommunikationstechnologien
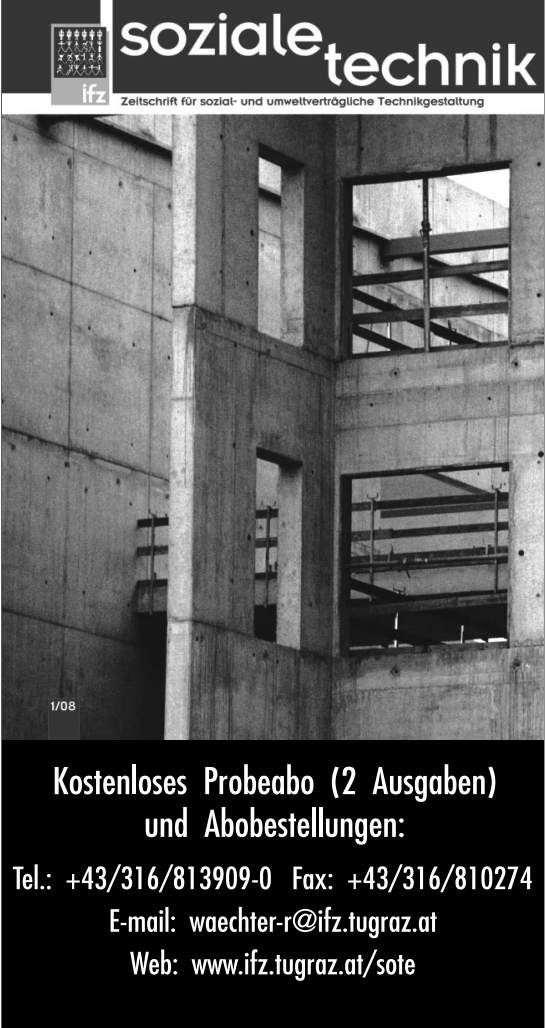
(c) 20I0 Authors; licensee IÖW and oekom verlag. This is an article distributed under the terms of the Creative Commons Attribution Non-Commercial No Derivates License (http://creativecommons.org/licenses/by-nc-nd/3.o/), which permits unrestricted use, distribution, and reproduction in any medium, provided the original work is properly cited. 